\title{
A Feasible Approach to Detect Pesticides in Food Samples Using THz-FDS and Chemometrics
}

\author{
Qian Chen, ${ }^{1}$ Shengyao Jia, ${ }^{1}$ Jianyuan Qin ${ }^{D},{ }^{1}$ Yong Du, ${ }^{1}$ and Zongshan Zhao ${ }^{2}$ \\ ${ }^{1}$ Center for Terahertz Research, China Jiliang University, Hangzhou 310018, China \\ ${ }^{2}$ College of Environmental Science and Engineering, Qingdao University, Qingdao 266071, China
}

Correspondence should be addressed to Jianyuan Qin; qinjianyuan44@163.com

Received 9 January 2020; Accepted 30 March 2020; Published 31 May 2020

Academic Editor: Wee Chew

Copyright (c) 2020 Qian Chen et al. This is an open access article distributed under the Creative Commons Attribution License, which permits unrestricted use, distribution, and reproduction in any medium, provided the original work is properly cited.

\begin{abstract}
The use of pesticides will have an impact on food, organisms, and environment. Specifically, pesticide residues in food will damage human health. Because of its high permeability, low energy, high spectral resolution, and fingerprint characteristics, terahertz frequency-domain spectroscopy has been introduced into the determination of pesticides (imidacloprid, acetamiprid, and triadimefon) residues in food samples (glutinous rice flour, wheat flour, and corn flour) in our present study. These three pesticides exhibit their own absorption peaks in the region of $0.4-1.7 \mathrm{THz}$. For understanding the origins of these peaks, the experimental data are interpreted by using density functional theory calculations at the level of B3LYP/6-31G (d). It is found that these absorption peaks come from the intramolecular and intermolecular interactions. The absorption peaks of pesticides are still detectable in a mixture of pesticides and food samples when they reach a certain concentration. The results from chemometrics analysis show that quantitative detection of pesticides in food samples is feasible. The partial least squares regression models have high correlation coefficient $(>0.99)$, low root-mean-square error of calibration $(<1.5 \%)$, low root-mean-square error of crossvalidation $(<2.4 \%)$, and low root-mean-square error of prediction $(<2.3 \%)$, indicating good quality of prediction for pesticides concentration. Our results prove that the terahertz frequency-domain spectrum combined with chemometrics can be used for the detection of pesticides in food samples.
\end{abstract}

\section{Introduction}

Imidacloprid, acetamiprid, and triadimefon, as common pesticides, are widely used in crop pest control and disease resistance. However, the irrational utilization of these pesticides can lead to unsafe residue levels in agricultural products, which will have potential harm for human health. In fact, pesticide residues in agricultural products are considered to be a huge health risk. Maximal residue limits of pesticides are established for different matrices by different countries. In the USA, the maximal residue limit of imidacloprid in wheat flour is $0.05 \mathrm{ppm}$ [1]. In China, the maximal residue limit of imidacloprid is $0.05 \mathrm{ppm}$, while the maximal residue limits of acetamiprid and triadimefon are both $0.5 \mathrm{ppm}$ [2]. However, the abusing of pesticides is becoming a serious problem despite the legal restrictions.
Therefore, it is of great significance to ensure the safety of agricultural products for the consumers.

In order to guarantee the safety of agricultural products, several detection methods have been proposed for successfully determination of pesticides in various matrices. The most commonly used method is gas chromatography-mass spectrometry. It has been applied to determinate the pesticides in wheat flour samples [3] and in pistachio samples [4], and it was found that these samples were within a safe range in terms of pesticide residue levels. This method has high sensitivity and good selectivity. However, the complex pretreatment of samples and the professional operation are needed in this method. Considering these deficiencies, the spectroscopy techniques, including infrared spectroscopy and Raman spectroscopy, have been introduced for the detection of pesticides [5]. These spectroscopy techniques 
have the advantages of nondestructive testing and simple operation, but they cannot detect the intermolecular vibrations of samples. Therefore, new spectroscopy methods are needed to detect the pesticide residues.

Terahertz $(\mathrm{THz})$ spectroscopy with a frequency band ranging from 0.1 to $10 \mathrm{THz}(30 \mu \mathrm{m}-3 \mathrm{~mm})$ is a type of electromagnetic radiation that locates between the microwave and infrared regions. Compared to other electromagnetic spectroscopy, $\mathrm{THz}$ spectroscopy has unique properties of high penetration, low ionization energy, and fingerprint characteristics [6, 7]. Due to its superiority, $\mathrm{THz}$ spectroscopy has been used as an analysis tool in many fields such as environmental detection [8], food analysis [9], and biomedical diagnosis $[10,11]$. In recent years, $\mathrm{THz}$ spectroscopy combined with chemometrics is increasingly used for qualitative and quantitative detection in the field of biochemistry [12]. THz time-domain spectroscopy ( $\mathrm{THz}$ TDS), a widely used $\mathrm{THz}$ technology, has been applied to the detection of glucose hydrochloric acid [13], amino acids $[14,15]$, and antibiotics [16]. It was found that these analytes all have fingerprint features in the $\mathrm{THz}$ range and thus their qualitative and quantitative detection can be achieved. In the field of pesticide detection, Chen et al. successfully detected imidacloprid in rice flour using THz-TDS [7]. Cao et al. used THz-TDS to investigate the coexistence of imidacloprid and carbendazim in flour and found that the absorption peaks of ternary mixtures were offset [17]. Compared with THz-TDS, $\mathrm{THz}$ frequency-domain spectroscopy (THz-FDS) based on photon mixing technology can provide a higher frequency resolution in a relatively wide spectral range [18]. In addition, the spectral information of the sample can be obtained without Fourier transform process as used in the case of THz-TDS [19]. Therefore, THz-FDS is considered to be a very promising analysis technology [20]. Wang et al. have reported the analysis of antibiotics in soil and chicken by THz-FDS and verified the practicable of this technology used for antibiotics detection in the matrix [16]. However, the application of THz-FDS is still in its infancy. So far, qualitative identification and quantitative analysis of pesticides by $\mathrm{THz}$-TDS combined with chemometrics have not been studied, and the origins of absorption peaks of imidacloprid, acetamiprid, and triadimefon have not been interpreted.

In this study, three pesticides including imidacloprid, acetamiprid, and triadimefon in food samples are detected using THz-FDS. It is found that these pesticides have their own absorption peaks. The results from density functional theory calculations show that the absorption peaks observed in the experiment come from the intramolecular and intermolecular interactions. The absorbance spectra of different concentrations of imidacloprid, acetamiprid, and triadimefon in glutinous rice flour are also investigated by using partial least squares regression algorithms. The results show that partial least squares regression models have good quality of prediction for pesticides concentration, with high correlation coefficient $(>0.99)$, low root-mean-square error of calibration $(<1.5 \%)$, low root-mean-square error of crossvalidation $(<2.4 \%)$, and low root-mean-square error of prediction $(<2.3 \%)$. The results in our present study verify the potential of THz-FDS as a tool for the detection of pesticide residues in food samples.

\section{Materials and Methods}

2.1. Materials. Pesticide samples, including imidacloprid (95\%), triadimefon (98\%), and acetamiprid (97\%), are purchased from Shanghai Runye Biological company (Shanghai, China). Glutinous rice flour, wheat flour, and corn flour are purchased from Huarun supermarket in Zhejiang Province, China. All these materials are used without further purification.

2.2. Sample Preparation. Pure pesticide samples contain only one pesticide. Binary mixture samples are the mixture of one pesticide in a food sample. Ternary mixture samples contain imidacloprid and triadimefon mixed with glutinous rice flour. The concentrations of pesticides in glutinous rice flour range from $0.5 \%$ to $50 \%(0.5 \%, 1 \%, 5 \%, 10 \%, 15 \%, 20 \%$, $25 \%, 30 \%, 40 \%$, and $50 \%$ ), while the concentration of pesticides in corn flour and wheat flour is set to $50 \%$. The $10 \%$ imidacloprid and 30\% triadimefon and 15\% imidacloprid and $20 \%$ triadimefon are mixed with glutinous rice flour to form ternary mixture samples, respectively. Then, each sample is weighed to a total mass of $200 \mathrm{mg}$ and placed in a circular tablet mold with a diameter of $13 \mathrm{~mm}$; a pressure of 6 tons is applied by using a tableting machine for 2 minutes. Three samples of each concentration are prepared, and the thickness of each sample is about $1 \mathrm{~mm}$. A total of 132 samples are prepared in our present study (18 for pure compound samples, 108 for binary mixture samples, and 6 for ternary mixture samples).

2.3. Spectral Measurements. A commercial THz-FDS (TeraScan 1550, TOPTICA Photonics AG, Germany) system is used to conduct the measurement of $\mathrm{THz}$ spectra, as shown in Figure 1. This system includes two distributed feedback (DFB) lasers, a $\mathrm{THz}$ transmitter, four off-axis parabolic mirrors, a sample holder, a $\mathrm{THz}$ receiver, and a signal processing system. THz waves are collimated and focused on the sample through the off-axis parabolic mirrors. $\mathrm{THz}$ waves propagate through the sample and are detected by the receiver. Samples containing imidacloprid and triadimefon are measured in the range of $0.4-1.1 \mathrm{THz}$, and samples containing acetamiprid are measured in the range of 0.4-1.2 THz. The reference spectra are collected when the $\mathrm{THz}$ waves passed through air, and they are collected before each sample measurement. Our experiment is carried out at room temperature with a relative humidity less than $30 \%$.

2.4. Spectral Analysis. THz waves are focused by the parabolic mirrors, and the photocurrent amplitude is obtained after scanning the sample. The absorbance of the sample can be calculated as follows: 


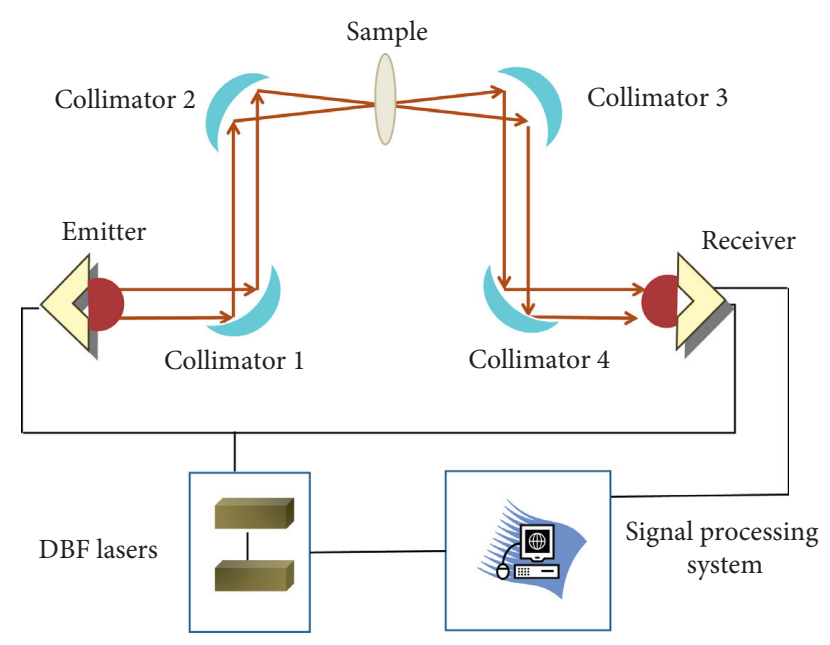

Figure 1: Schematic diagram of THz-FDS system.

$$
\alpha=-\lg T,
$$

where $T$ is the transmittance, which is calculated by comparing the photocurrent amplitude of sample to that of reference. The calculations of absorbance spectra are carried out by using the TOPAS_cw Terahertz Control software (version 2.5.0.1870, TOPTICA Photonics AG, Germany) and the Origin software (version 8.0724, Origin Lab Inc. USA).

2.5. Density Functional Theory. In order to interpret the origins of absorption peaks for these pesticides, the experimental data are interpreted using density functional theory (DFT) calculations by considering a single molecule of the pesticides. DFT is a quantum mechanics method for studying the electronic structure of multielectronic systems by taking into account the correlative effects of electron energy and spin. It is suitable for the analysis of most molecular structures with high accuracy and low computational complexity [21]. B3LYP hybrid density function and 6-31G (d) basis set are used for DFT calculations, where (d) denotes the addition of polarization functions made by non$\mathrm{H}$ atoms [21]. The effectiveness of the combination of the B3LYP/6-31G (d) for interpreting the $\mathrm{THz}$ spectrum has been demonstrated previously [22]. The DFT calculations are carried out in Gaussian 09 software (revision D.01, Gaussian Inc., USA) [23].

2.6. Chemometrics Analysis. Chemometrics have become an important tool for qualitative and quantitative analysis in the spectroscopic technology. Partial least squares regression (PLSR) is used to establish the prediction model $[24,25]$ in our study. The low root-mean-square error of calibration (RMSEC), low root-mean-square error of prediction (RMSEP), low root-mean-square error of cross-validation (RMSECV), and correlation coefficient $(R)$ values are used to evaluate the model performance [25-28]. In each model, the data of all bands are used for modeling. In order to improve the accuracy of model, the spectra are preprocessed using the
Savitzky-Golay (S-G) smoothing method [29] and the multiscatter correction (MSC) [30]. The S-G smoothing method is typically used to smooth out the noise signal in the spectrum, while MSC is used to eliminate the influence of sample scattering. The simple linear regression (SLR) model is applied to describe the relationship between pesticide concentration in glutinous rice flour and absorbance. The coefficient of determination $\left(R^{2}\right)$ that represents the degree of influence of independent variables on dependent variable is used to evaluate the prediction performance of the model. The higher the $R^{2}$ value is, the more accurate the prediction is [22]. The chemometrics are realized by using the TQ analysts (version 8.3.0.125, Thermo Fisher Scientific Inc., USA) software.

\section{Results and Discussion}

3.1. Qualitative Analysis. Figure 2 shows the measured absorbance spectra of imidacloprid, acetamiprid, and triadimefon in the range of $0.4-1.7 \mathrm{THz}$ (black lines) and the calculated discrete spectra of corresponding pesticide molecule (red lines). It can be seen from Figure 2(a) that imidacloprid has a narrow and distinct absorption peak at $0.88 \mathrm{THz}$, two less obvious absorption peaks at $1.14 \mathrm{THz}$ and $1.27 \mathrm{THz}$, and a relatively obvious and broad absorption peak at $1.49 \mathrm{THz}$. The most remarkable absorption peak is observed at $0.88 \mathrm{THz}$, which agrees well with the previous report $[7,17]$. In the calculation, imidacloprid has an absorption peak at $1.08 \mathrm{THz}$, which corresponds to the peak at $1.14 \mathrm{THz}$ in the measurements. This peak is mainly caused by the joint motion of two parts: one is the deformation and tension vibration of pyridine ring and the other is the torsion of hydrogen atom, as shown in Figure 3(a). Figure 2(b) shows that acetamiprid has two obvious and relatively broad absorption peaks at $1.08 \mathrm{THz}$ and $1.44 \mathrm{THz}$. Calculations show that there are two absorption peaks at $0.98 \mathrm{THz}$ and $1.4 \mathrm{THz}$ for acetamiprid, which correspond to the peaks at $1.08 \mathrm{THz}$ and $1.44 \mathrm{THz}$ in the measurements, respectively. The peak at $0.98 \mathrm{THz}$ is caused by the torsion of methylene, methyl, and carbon atoms, while the peak at $1.4 \mathrm{THz}$ comes from the twisting of methylene and hydrogen atoms, as shown in Figures 3(b) and 3(c). The triadimefon has three obvious and narrow absorption peaks at $0.95 \mathrm{THz}, 1.18 \mathrm{THz}$, and $1.62 \mathrm{THz}$ (Figure 2(c)). Calculations show that there exists an absorption peak at $0.91 \mathrm{THz}$ caused by the tensile vibration of the fused heterocycle (Figure 3(d)), which corresponds to the peak at $0.95 \mathrm{THz}$ in the measurements. These results demonstrate that these three pesticides have their own distinct $\mathrm{THz}$ absorption peaks that can be used for qualitative analysis. The absorption peak at $1.14 \mathrm{THz}$ for imidacloprid, peaks at $1.08 \mathrm{THz}$ and $1.44 \mathrm{THz}$ for acetamiprid, and peak at $0.95 \mathrm{THz}$ for triadimefon are derived from the intramolecular interactions and can be observed in the theoretical calculations. The slight differences in the intensity and the position of absorbance peaks between the measurements and calculations are due to the fact that the experiments are carried out at room temperature while the calculations are at 0 degrees. Other absorption peaks that do not appear in the calculation are considered as a result of 


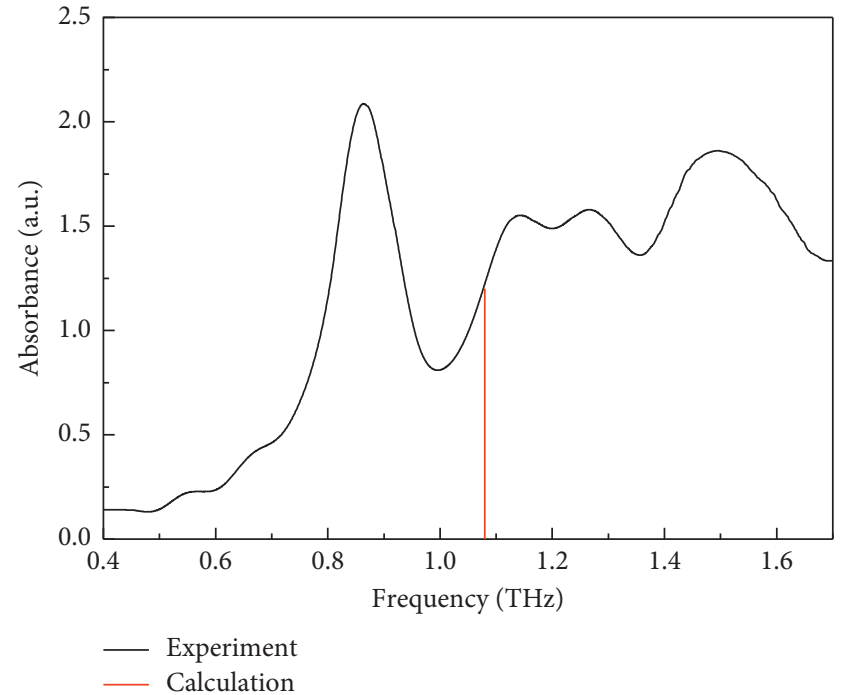

(a)

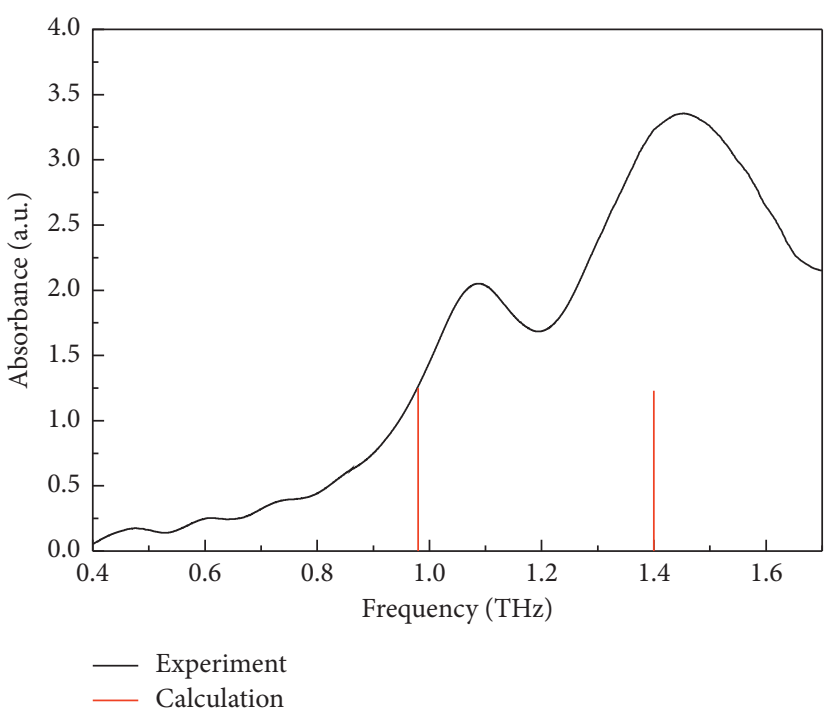

(b)

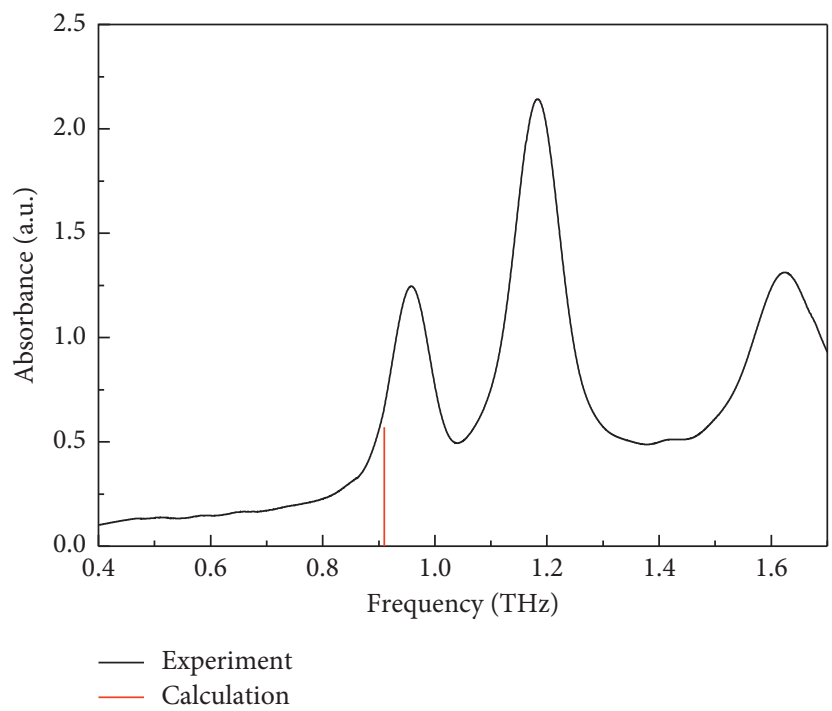

(c)

FIGURE 2: Measured absorbance spectra (black lines) and calculated discrete spectra (red lines) of the imidacloprid (a), acetamiprid (b), and triadimefon $(c)$.

intermolecular interactions. Because the calculations are based on the single molecule structure, thus no intermolecular interactions are shown in their results.

Figure 4 shows the absorbance spectra of glutinous rice flour, wheat flour, and corn flour in the $0.4-1.2 \mathrm{THz}$. It is found that glutinous rice flour has no distinct absorption peaks in this band, as reported in the previous study [7]. Similarly, there are no absorption peaks in the absorbance spectra of wheat flour and corn flour. Corn flour has a relatively high absorbance ranging from 0.72 to 3.2. In the region of $0.61-1.2 \mathrm{THz}$, the absorbance spectra of glutinous rice flour and wheat flour are overlapping with an absorbance ranging from 1.18 to 2.94 , while in $0.4-0.61 \mathrm{THz}$, the absorbance spectrum of glutinous rice flour is higher than that of corn flour, with the absorbance ranging from 0.76 to 1.18. In addition, a slope background is observed in these absorbance spectra. This is because there are various components in these food samples such as cellulose, protein, and starch that can absorb $\mathrm{THz}$ radiation and thus cause scattering. These results indicate that the pesticides mentioned above are likely identified in these food samples according to their fingerprint features.

3.2. Quantitative Analysis. The absorbance spectra of binary mixture samples in the $0.4-1.2 \mathrm{THz}$ region are investigated to explore the possibility of THz-FDS for quantitative detection of pesticides in the food samples. The absorbance spectra of imidacloprid, acetamiprid, and triadimefon in glutinous rice flour at different concentrations are analyzed in detail, as shown in Figure 5. Besides, a simple analysis of the absorbance spectra of these pesticides in wheat flour and 


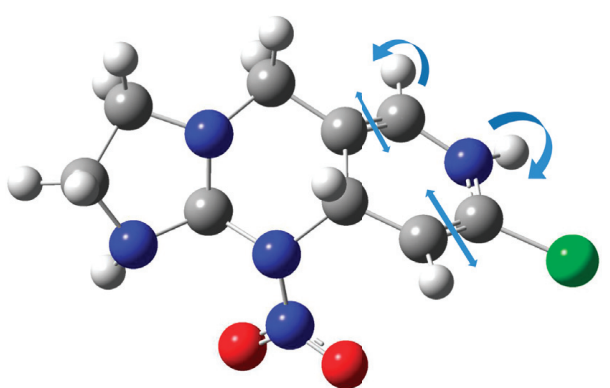

(a)

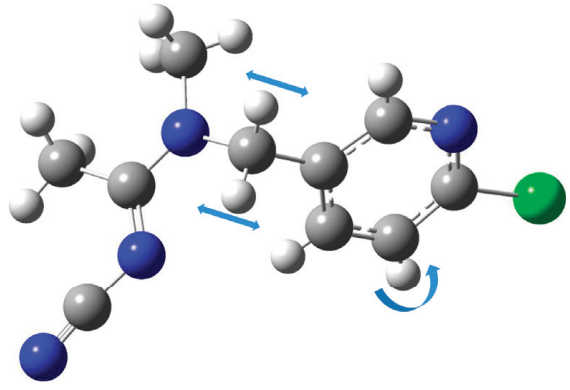

(c)

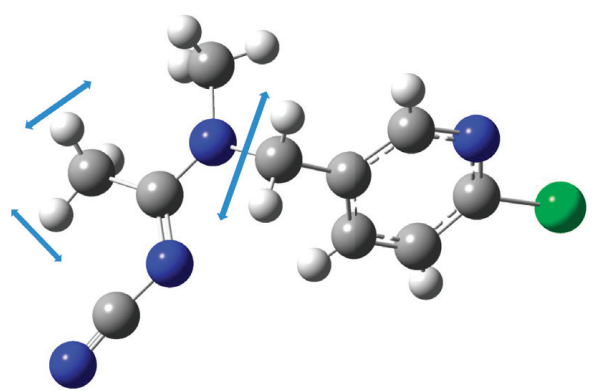

(b)

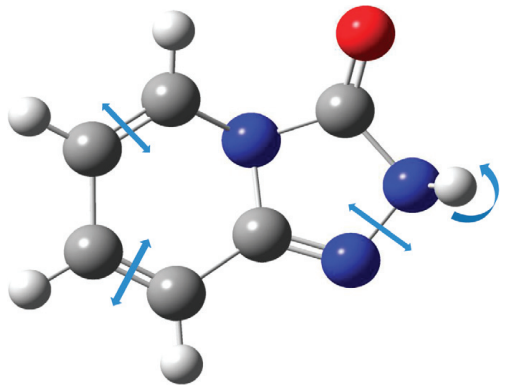

(d)

Figure 3: Calculated vibration modes of (a) imidacloprid at $1.08 \mathrm{THz},(\mathrm{b})$ acetamiprid at $0.98 \mathrm{THz}$, (c) acetamiprid at $1.4 \mathrm{THz}$, and (d) triadimefon at $0.91 \mathrm{THz}$.

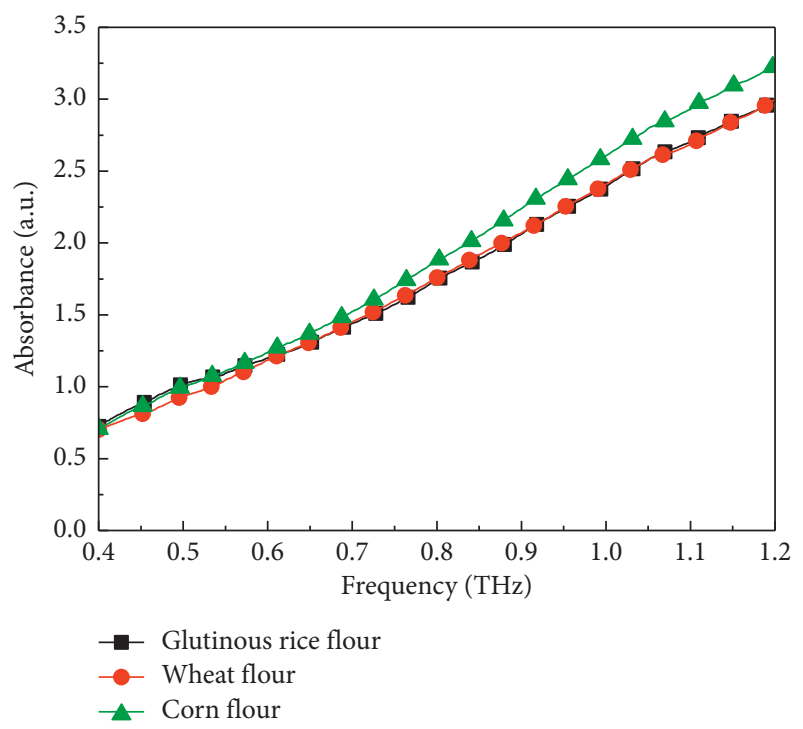

FIGURE 4: Measured absorbance spectra of glutinous rice flour, wheat flour, and corn flour.

corn flour is also presented (the insets in Figure 5). Figure 5(a) shows the absorbance spectra of imidacloprid in glutinous rice flour with different concentrations. The absorption peak of imidacloprid at $0.88 \mathrm{THz}$ can be clearly identified when the imidacloprid concentration is greater than $5 \%$. As the concentration increases, the absorption peak becomes more and more obvious. When the imidacloprid concentration is lower than $1 \%$, no significant absorption peak can be identified. The absorbance spectra of the imidacloprid in the corn flour and wheat flour at the concentration of $50 \%$ are also plotted in the inset in Figure 5(a). The absorption peak of imidacloprid at $0.88 \mathrm{THz}$ can be clearly observed. In the case of acetamiprid, the peak at $1.08 \mathrm{THz}$ can be distinguished when the concentration is greater than $10 \%$ as shown in Figure 5(b). The absorbance of acetamiprid is comparable to that of glutinous rice flour, as shown in the Figures 2(b) and 4 (the red line with circles). Therefore, the absorbance of glutinous rice flour has an important impact on the absorbance spectra of their binary mixture samples, and no absorption peak appeared when the acetamiprid concentration is lower than $10 \%$. In addition, there are no regular changes of the absorbance with the increase of acetamiprid concentration. The inset in the Figure 5(b) shows that the absorption peak of acetamiprid can also be detected in both cases of corn flour and wheat flour at the concentration of 50\%. However, for triadimefon, the absorbance decreases, but the absorption peak at $0.95 \mathrm{THz}$ becomes more and more obvious as the concentration increases, as shown in Figure 5(c). When the triadimefon concentration is greater than $5 \%$, its absorption peak can be identified easily. Although the absorbance spectra of triadimefon with a concentration of $0.5 \%$ and $1 \%$ had no distinct absorption peak, they could be distinguished from each other based on the differences in the absorbance. The inset in Figure 5(c) shows that the absorption peak of triadimefon with a concentration of $50 \%$ can be detected clearly when mixed with corn flour and wheat flour, respectively.

The analysis for absorbance spectra of these three pesticides in glutinous rice flour indicated that these pesticides are still identifiable when mixed with glutinous rice flour. When the concentration falls below a certain level, the 


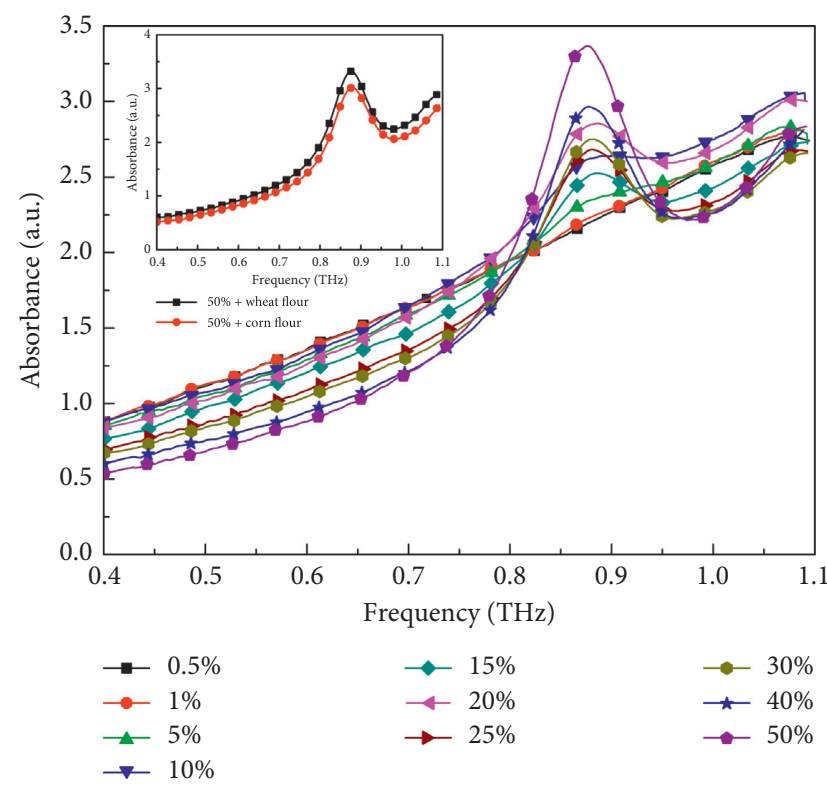

(a)

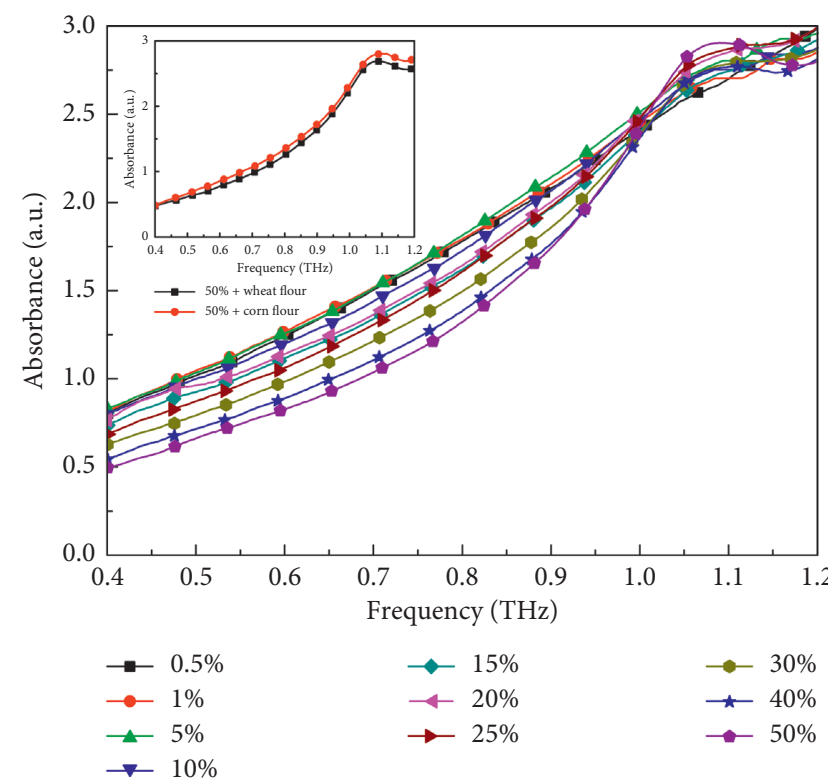

(b)

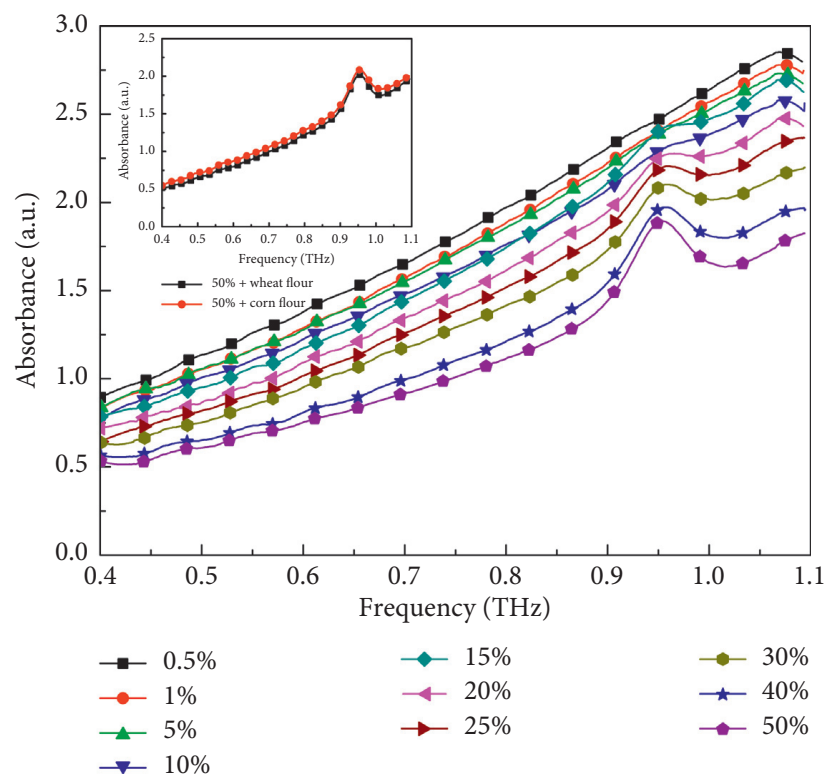

(c)

Figure 5: Measured absorbance spectra of imidacloprid (a), acetamiprid (b), and triadimefon (c) in the glutinous rice flour with different concentrations. The insets show the corresponding pesticides with a concentration of $50 \%$ in corn flour and wheat flour.

absorption peaks of pesticides cannot be clearly identified or even disappeared. This phenomenon can be explained by the fact that the absorbance of glutinous rice flour plays a dominant role in the absorbance spectra, which masks the absorption peaks of pesticides. However, although without the absorption peaks at low concentrations, each concentration of pesticides can be distinguished from each other based on the differences in the absorbance. The absorbance spectra of these three pesticides with a concentration of 50\% in corn flour and wheat flour are given as an example to verify the feasibility of detecting pesticides in other food samples using THz-FDS.
To further evaluate the performance of THz-FDS used for detecting these pesticides in glutinous rice flour, the PLSR models for these absorbance spectra are established. Each model contained 30 binary mixture samples with 10 concentrations $(0.5 \%, 1 \%, 5 \%, 10 \%, 15 \%, 20 \%, 25 \%, 30 \%$, $40 \%$, and $50 \%$ ). For each model, 23 binary mixture samples with 10 concentrations $(0.5 \%, 1 \%, 5 \%, 10 \%, 15 \%, 20 \%, 25 \%$, $30 \%, 40 \%$, and $50 \%$ ) are used as the calibration set, and 7 binary mixture samples with 7 concentrations $(5 \%, 10 \%$, $15 \%, 20 \%, 25 \%, 30 \%$, and $40 \%$ ) are used as the prediction set. The results of the PLSR models are plotted in Figure 6 and their corresponding model performance is summarized in 


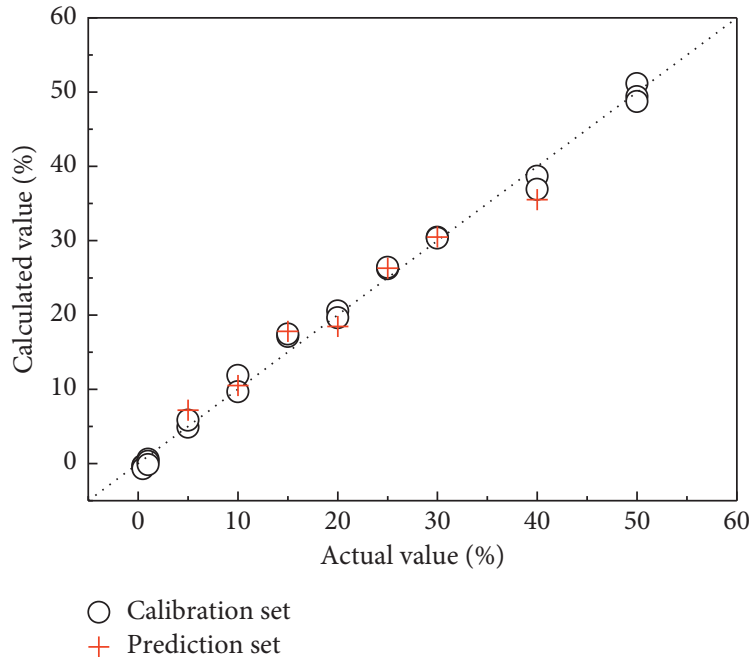

(a)

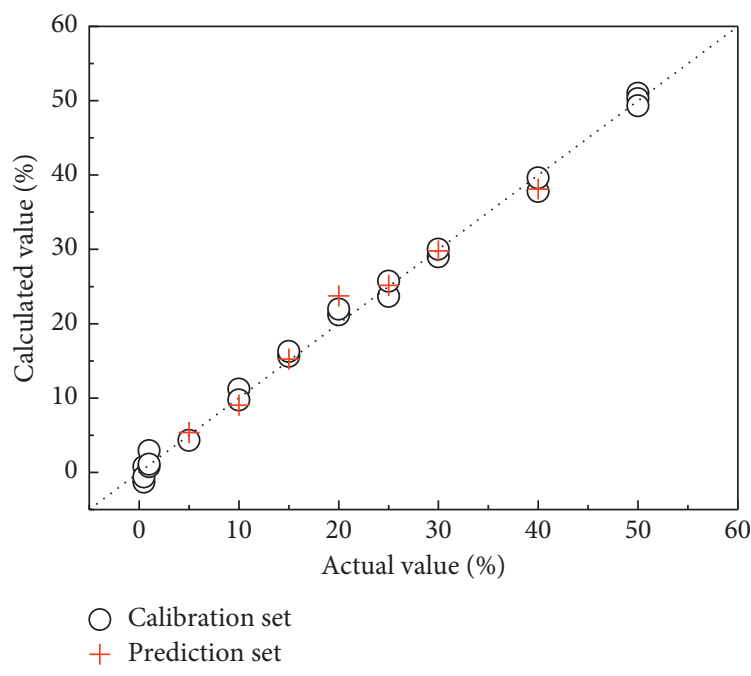

(c)

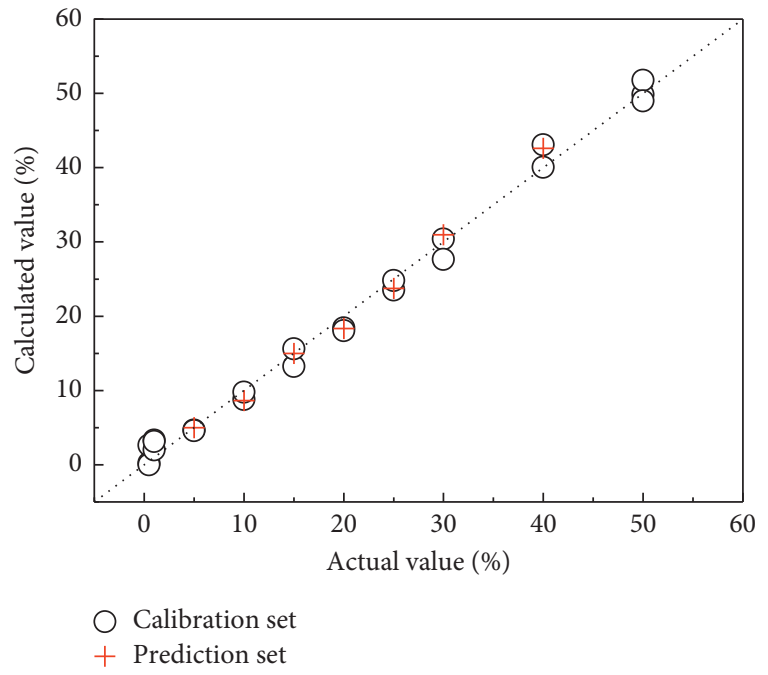

(e)

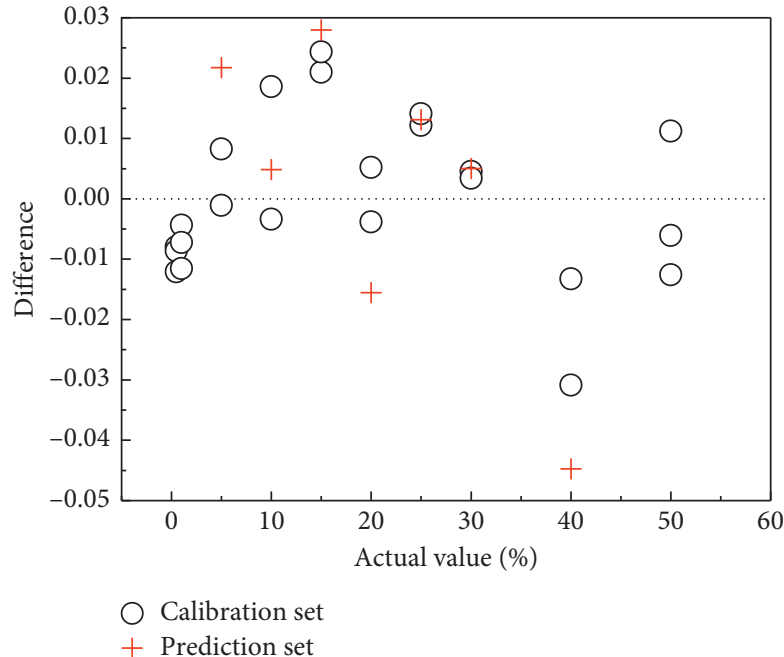

(b)

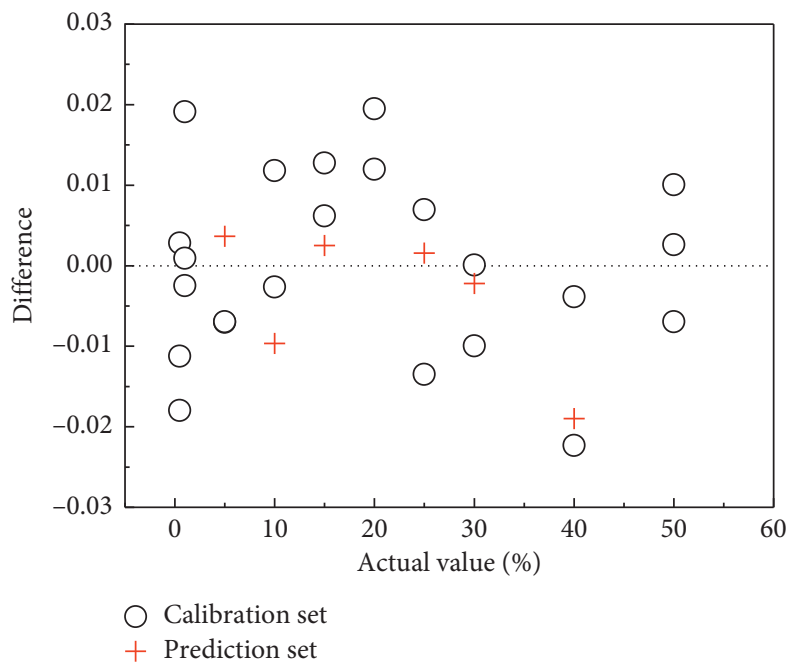

(d)

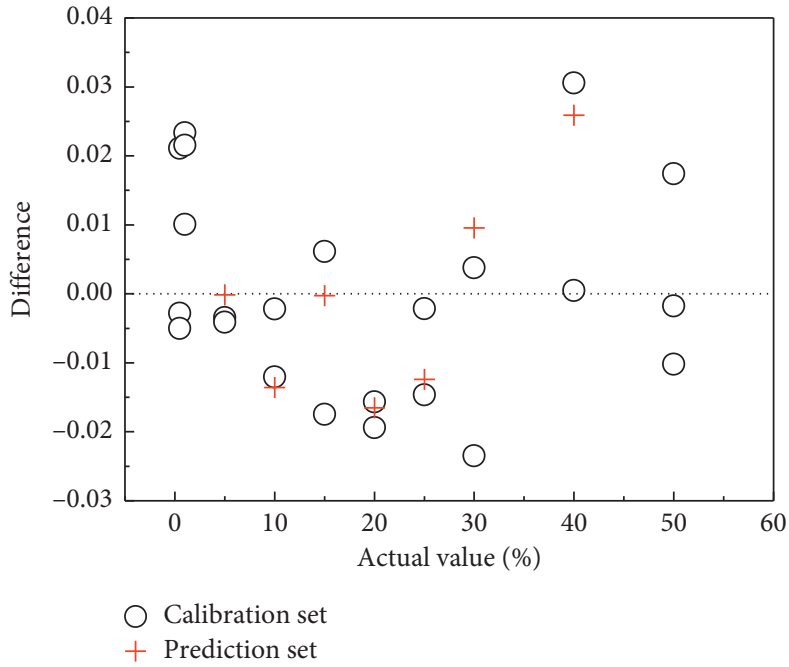

(f)

FIGURE 6: Correlation statistics between the actual values and calculated values of imidacloprid (a), acetamiprid (c), and triadimefon (e). Error statistics of actual value and calculated value of imidacloprid (b), acetamiprid (d), and triadimefon (f). 
TABle 1: Descriptive statistics of the prediction models built by PLSR.

\begin{tabular}{lccccc}
\hline Model & RMSEC (\%) & RMSEP (\%) & RMSECV (\%) & $R$ & Factors \\
\hline Imidacloprid & 1.3 & 2.3 & 2.4 & 0.997 & 4 \\
Acetamiprid & 1.1 & 1.6 & 1.5 & 0.998 & 2 \\
Triadimefon & 1.5 & 1.4 & 2.2 & 0.997 \\
\hline
\end{tabular}

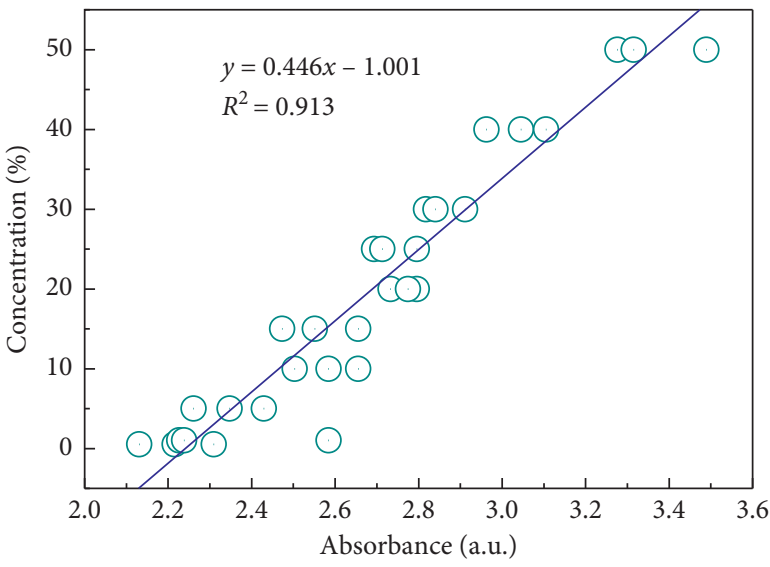

(a)

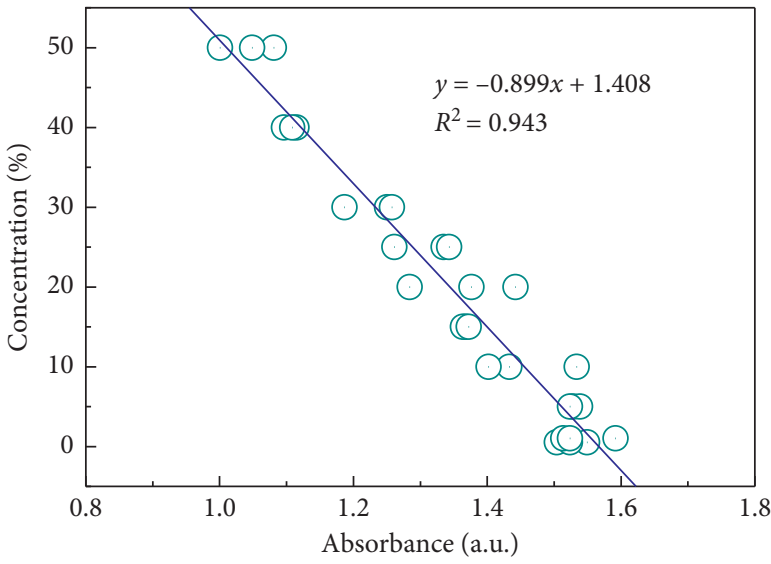

(b)

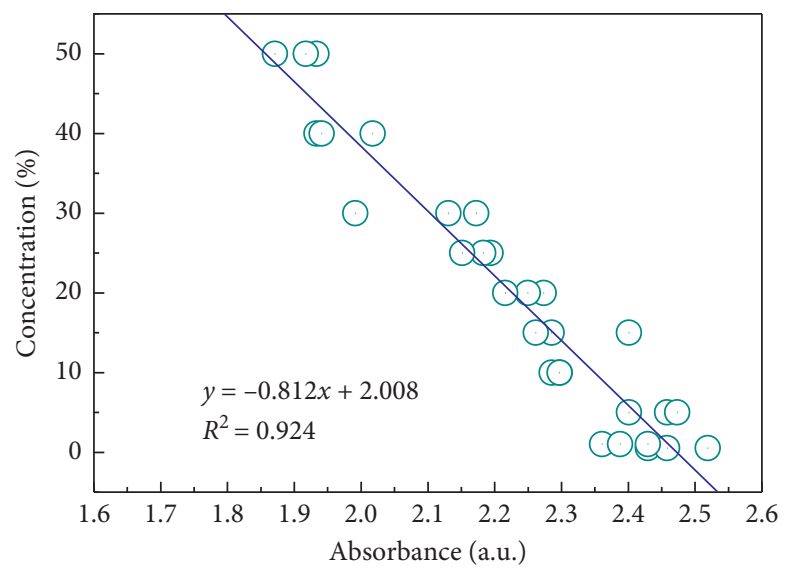

(c)

FIGURE 7: SLR models of (a) imidacloprid, (b) acetamiprid, and (c) triadimefon in the glutinous rice flour.

Table 1. Figures 6(a), 6(c), and 6(e) show the correlation statistics between the actual values and calculated values of these three pesticides in glutinous rice flour. It can be found that all the data points are distributed very close to the diagonal dotted line, indicating the high accuracy of these models. Figures 6(b), 6(d), and 6(f) show the error in concentration in these models. The calibration set and the prediction set in each model are close to the error value of 0 , and the difference between the actual values and calculated values is less than 0.05 , which confirm the credibility of PLSR for the quantitative analysis of pesticides. Good performance is obtained for these PLSR models, with high correlation coefficient value $(>0.99)$ and low root-mean-square error value $(<2.4 \%)$. The acetamiprid model performs the best with $R=0.99, \quad$ RMSEC $=1.1 \%$, $\operatorname{RMSECV}=1.5 \%$, and RMSEP $=1.6 \%$. The imidacloprid model comes second with
$R=0.99, \quad$ RMSEC $=1.3 \%, \quad$ RMSECV $=2.4 \%, \quad$ and RMSEP $=2.3 \%$, while the triadimefon model comes in last with $R=0.99, \quad \mathrm{RMSEC}=1.5 \%, \quad \mathrm{RMSECV}=2.2 \%$, and RMSEP $=1.4 \%$. These results indicate that these PLSR models are effective and reliable for prediction, highlighting the potential of $\mathrm{THz}-\mathrm{FDS}$ combined with chemometrics analysis to the detection of pesticide residues in food samples.

The SLR models for describing the relationship between pesticide concentration in glutinous rice flour and absorbance are shown in Figure 7. Figure 7(a) shows the SLR model for the concentration imidacloprid in glutinous rice flour and the absorbance at $0.88 \mathrm{THz}$. The $R^{2}$ value is 0.913 , indicating a good linear regression relationship. Figure 7 (b) shows the SLR model of acetamiprid concentration in glutinous rice flour and the absorbance at $0.7 \mathrm{THz}$, the $R^{2}$ 


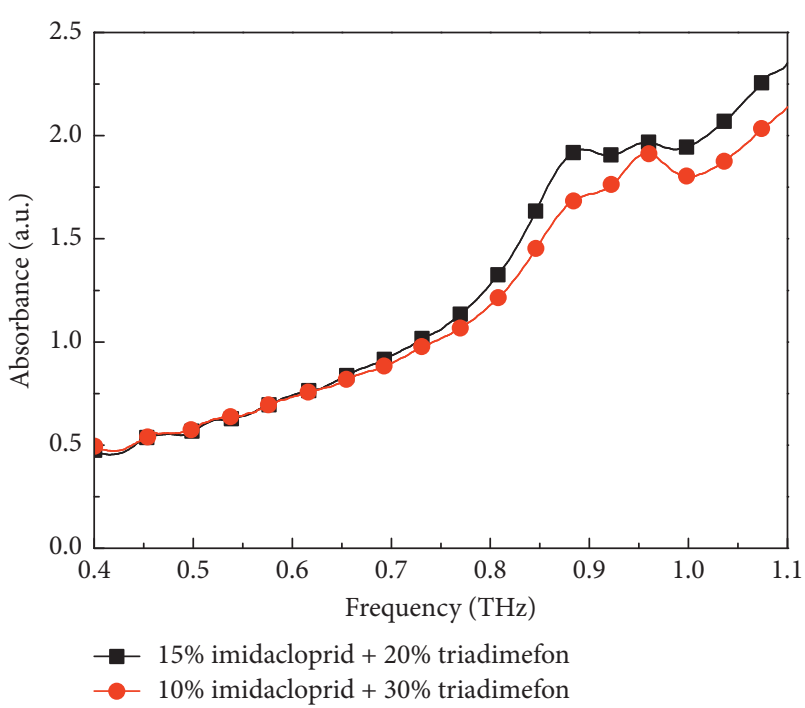

FIGURE 8: Measured absorbance spectra of ternary mixture samples at different concentrations.

value reaches 0.943 . Figure $7(\mathrm{c})$ presents the SLR model between the triadimefon concentration in glutinous rice flour and the absorbance at $0.95 \mathrm{THz}$, resulting in an $R^{2}$ value of 0.924 . All these models have the $R^{2}$ reaching above 0.91 , demonstrating the great potential of $\mathrm{THz}$ technology combined with SLR model for pesticide detection in glutinous rice flour.

3.3. Analysis of Ternary Mixture Samples. In order to detect the simultaneous presence of various pesticides in glutinous rice flour, ternary mixture samples of glutinous rice flour containing two different pesticides are investigated. Imidacloprid and triadimefon are selected as the targets because they contain remarkable absorption peaks located at different frequencies (imidacloprid at $0.88 \mathrm{THz}$ and triadimefon at $0.95 \mathrm{THz}$ ). Figure 8 shows the absorbance spectra of these ternary mixture samples. In the case where $15 \%$ imidacloprid and $20 \%$ triadimefon are added to the glutinous rice flour, the absorption peak of imidacloprid at $0.88 \mathrm{THz}$ is observed, while the absorption peak of triadimefon at $0.95 \mathrm{THz}$ is not obvious. This is because the absorbance at the absorption peak of triadimefon is much lower than that of imidacloprid. When the $10 \%$ imidacloprid and $30 \%$ triadimefon are added to the glutinous rice flour, the absorption peak of triadimefon at $0.95 \mathrm{THz}$ is highlighted. At the same time, the absorption peak of imidacloprid at $0.88 \mathrm{THz}$ can also be clearly observed. The absorbance of the sample containing 10\% imidacloprid and $30 \%$ triadimefon is lower than that of the sample containing $15 \%$ imidacloprid and 20\% triadimefon. The reasons for this are that the absorbance of glutinous rice flour and imidacloprid is higher than that of triadimefon, and the content of glutinous rice flour and imidacloprid in the former is higher than that in the latter, resulting in an overall decrease of absorbance. The above results preliminarily indicate that THz-FDS can be used to detect two different pesticides in glutinous rice flour.

\section{Conclusion}

In this study, a new method based on THz-FDS combined with chemometrics is developed for the detection of imidacloprid, acetamiprid, and triadimefon in food samples. By comparing the experimental results and DFT calculations, it is verified that the absorption peaks of these pesticides come from intramolecular and intermolecular interaction. PLSR models are used to realize the quantitative analysis of these pesticides in glutinous rice flour and demonstrate that these models have the high $R(>0.99)$ and low RMSEC $(<1.5 \%)$, RMSECV $(<2.4 \%)$, and RMSEP $(<2.3 \%)$. SLR models that describe the relationship between pesticide concentration in glutinous rice flour and absorbance have the high $R^{2}(>0.91)$. The results show that this method can reliably predict the concentration of pesticides in food samples. At the same time, this method can detect two different pesticides in the ternary mixture samples. Our study indicates that THz-FDS combined with chemometrics can be utilized for pesticide detection in food samples.

\section{Data Availability}

The data used to support the findings of this study are available from the corresponding author upon request.

\section{Conflicts of Interest}

The authors declare that there are no conflicts of interest.

\section{Acknowledgments}

This work was supported by the financial support from the National Natural Science Foundation of China (NSFC) (Grant no. 61705213) and the Natural Science Foundation of Zhejiang Province (Grant no. LY19B050003).

\section{References}

[1] J. Sun, "Extraction and analysis method of residual imidacloprid in vegetables," Modem Instruments \& Medical, vol. 15, no. 1, pp. 5-10, 2009.

[2] National Food Safety Standard-Maximum Residue Limits for Pesticides in Food, GB2763-2016, 2016.

[3] A. Emami, Z. Mousavi, V. Ramezani et al., "Residue levels and risk assessment of pesticides in pistachio nuts in Iran," Iranian Journal of Toxicology, vol. 11, no. 2, 2017.

[4] M. Rezaei, N. Shariatifar, S. Shoeibi, M. Jahed Khaniki, and G. J. Khaniki, "Simultaneous determination of residue from 58 pesticides in the wheat flour consumed in Tehran, Iran by GC/MS," Iranian Journal of Pharmaceutical Research: IJPR, vol. 16, no. 3, pp. 1048-1058, 2017.

[5] S. Buckingham, B. Lapied, H. Corronc, and F. Sattelle, "Imidacloprid actions on insect neuronal acetylcholine receptors," The Journal of Experimental Biology, vol. 200, no. 21, pp. 2685-2692, 1997.

[6] K. Müller, A. Tiktak, T. J. Dijkman, S. Green, and B. Clothier, "Advances in pesticide risk reduction," Encyclopedia of Agriculture and Food Systems, pp. 17-34, Academic Press, Cambridge, MA, USA, 2014.

[7] Z. Chen, Z. Zhang, R. Zhu, Y. Xiang, Y. Yang, and P. B. Harrington, "Application of terahertz time-domain 
spectroscopy combined with chemometrics to quantitative analysis of imidacloprid in rice samples," Journal of Quantitative Spectroscopy and Radiative Transfer, vol. 167, pp. 1-9, 2015.

[8] V. L. Vaks, S. I. Pripolsin, A. N. Panin et al., "Towards sub ppq detection and monitoring capabilities for environmental applications using novel $\mathrm{THz}$ technologies," in International Conference on Infrared, Millimeter, and Terahertz Waves, IEEE, Houston, TX, USA, December 2011.

[9] C. Du, X. Zhang, and Z. Zhang, "Quantitative analysis of ternary isomer mixtures of saccharide by terahertz time domain spectroscopy combined with chemometrics," $V i$ brational Spectroscopy, vol. 100, pp. 64-70, 2019.

[10] Y. Peng, X. Yuan, X. Zou et al., "Terahertz identification and quantification of neurotransmitter and neurotrophy mixture," Biomedical Optics Express, vol. 7, no. 11, pp. 4472-4479, 2016.

[11] P. Bawuah, J. A. Zeitler, J. Ketolainen, and K.-E. Peiponen, "Terahertz absorption spectra of commonly used antimalarial drugs," Optical Review, vol. 25, no. 3, pp. 444-449, 2018.

[12] Y. Peng, C. Shi, M. Xu et al., "Qualitative and quantitative identification of components in mixture by terahertz spectroscopy," IEEE Transactions on Terahertz Science and Technology, vol. 8, no. 6, pp. 696-701, 2018.

[13] S. Li, J. Yang, H. Zhao et al., "Terahertz time-domain spectroscopy and quantitative analysis of metal gluconates," Applied Spectroscopy, vol. 69, no. 1, pp. 52-57, 2015.

[14] X. Zhang, S. Lu, Y. Liao, and Z. Zhang, "Simultaneous determination of amino acid mixtures in cereal by using terahertz time domain spectroscopy and chemometrics," Chemometrics and Intelligent Laboratory Systems, vol. 164, pp. 8-15, 2017.

[15] S. H. Lu, B. Q. Li, H. L. Zhai, X. Zhang, and Z. Y. Zhang, "An effective approach to quantitative analysis of ternary amino acids in foxtail millet substrate based on terahertz spectroscopy," Food Chemistry, vol. 246, pp. 220-227, 2018.

[16] Y. Wang, Q. Wang, Z. Zhao, A. Liu, Y. Tian, and J. Qin, "Rapid qualitative and quantitative analysis of chlortetracycline hydrochloride and tetracycline hydrochloride in environmental samples based on terahertz frequency-domain spectroscopy," Talanta, vol. 190, pp. 284-291, 2018.

[17] B. Cao, H. Li, M. Fan, W. Wang, and M. Wang, "Determination of pesticides in a flour substrate by chemometric methods using terahertz spectroscopy," Analytical Methods, vol. 10, no. 42, pp. 5097-5104, 2018.

[18] H. Zhang, Z. H. Zhang, X. Y. Zhao et al., "Qualitative and quantitative analysis of atmospheric methanol using a continuous-wave terahertz spectrometer," Chinese Optics Letters, vol. 16, no. 10, Article ID 103001, 2018.

[19] M. Yahyapour, N. Vieweg, A. Roggenbuck, F. Rettich, O. Cojocari, and A. Deninger, "A flexible phase-insensitive system for broadband CW-terahertz spectroscopy and imaging," IEEE Transactions on Terahertz Science and Technology, vol. 6, no. 5, pp. 670-673, 2016.

[20] S. Brinkmann, N. Vieweg, G. Gärtner, P. Plew, and A. Deninger, "Towards quality control in pharmaceutical packaging: screening folded boxes for package inserts," Journal of Infrared Millimeter \& Terahertz Waves, vol. 38, pp. 339-346, 2016.

[21] P. Nie, C. Cai, F. Qu et al., "Study of 2, 4-D spectral characteristics and its detection in zizania latifolia using terahertz time-domain spectroscopy," Applied Sciences, vol. 9, no. 11, 2019.
[22] Q. Miao, L. Tian, K. Zhao et al., "Detection and identification of selected alcohols using terahertz time-domain spectroscopy," Journal of Physics: Conference Series, vol. 276, Article ID 012220, 2011.

[23] M. J. Frisch, G. W. Trucks, H. B. Schlegel et al., Gaussian 09, Revision D. 01, Gaussian, Inc., Wallingford CT, USA, 2009.

[24] Y. Hua and H. Zhang, "Qualitative and quantitative detection of pesticides with terahertz time-domain spectroscopy," IEEE Transactions on Microwave Theory and Techniques, vol. 58, no. 7, pp. 2064-2070, 2010.

[25] J. B. Sleiman, B. Bousquet, N. Palka, and P. Mounaix, "Quantitative analysis of hexahydro-1, 3, 5-trinitro-1, 3, 5, triazine/pentaerythritol tetranitrate (RDX-PETN) mixtures by terahertz time domain spectroscopy," Applied Spectroscopy, vol. 69, no. 12, pp. 1464-1471, 2015.

[26] W. Qiang and Y. H. Ma, "Qualitative and quantitative identification of nitrofen in terahertz region," Chemometrics and Intelligent Laboratory Systems, vol. 127, pp. 43-48, 2013.

[27] H. Zhang, Z. Li, T. Chen, and J. Liu, "Discrimination of traditional herbal medicines based on terahertz spectroscopy," Optik, vol. 138, pp. 95-102, 2017.

[28] J. Qin, L. Xie, and Y. Ying, "Determination of tetracycline hydrochloride by terahertz spectroscopy with PLSR model," Food Chemistry, vol. 170, pp. 415-422, 2015.

[29] P. U. Jepsen and B. M. Fischer, "Dynamic range in terahertz time-domain transmission and reflectionspectroscopy," $O p$ tics Letters, vol. 30, no. 1, pp. 29-31, 2005.

[30] E. Teye, C. L. Y. Amuah, T. McGrath, and C. Elliott, "Innovative and rapid analysis for rice authenticity using handheld NIR spectrometry and chemometrics," Spectrochimica Acta Part A: Molecular and Biomolecular Spectroscopy, vol. 217, pp. 147-154, 2019. 